\title{
ON PRODUCTS OF POWERS IN GROUPS
}

\author{
ROGER LYNDON, ${ }^{1}$ THOMAS MCDONOUGH AND MORRIS NEWMAN
}

ABSTRACT. In this note we show that a product of $N$ th powers in a group cannot in general be expressed as a product of fewer $N$ th powers. This extends a result of Lyndon and Newman [1].

THEOREM. Let $F$ be a free group of rank $n$ with basis $x_{1}, \cdots, x_{n}$, let $u_{1}, \cdots, u_{m}$ be elements of $F$, and let $N$ be an integer greater than 1 . If

$$
x_{1}^{N} \cdots x_{n}^{N}=u_{1}^{N} \cdots u_{m}^{N}
$$

then $m \geqq n$.

For the proof it will suffice to exhibit a group $G$ and elements $x_{1}, \cdots, x_{n}$ in $G$ such that, if $u_{1}, \cdots, u_{m}$ are any elements of $G$ satisfying (*), then $m \geqq n$.

Choose a prime $p$ dividing $N$ and write $N=q M$, where $q=p^{e}$ for some $e \geqq 1$ and $p$ does not divide $M$. Let $P$ be the ring of polynomials over $G \bar{F}(p)$ in noncommuting indeterminates $X_{1}, \cdots, X_{n}$. Let $\mathscr{J}$ be the ideal in $P$ generated by $X_{1}, \cdots, X_{n}$, and let $R=P / \mathscr{J}^{q+1}$; we shall write $X_{i}$ also for the image of $X_{i}$ in $R$. Let $G$ be the group of units in $R$. (Thus $G$ is a finite group of exponent pq.) The elements $x_{i}=1+X_{i}$ belong to $G$, since they have inverses $x_{i}^{-1}=1-X_{i}+X_{i}^{2}-\cdots+(-1)^{q} X_{i}^{q}$.

Now $x_{i}^{q}=\left(1+X_{i}\right)^{q}=1+X_{i}^{q}$, whence $x_{i}^{N}=x_{i}^{q M}=\left(1+X_{i}^{q}\right)^{M}=1+M x_{i}^{q}$. It follows that

$$
x_{1}^{N} \cdots x_{n}^{N}=1+M \sum_{i=1}^{n} x_{i}^{q} .
$$

Let $u_{1}, \cdots, u_{m}$ be in $G$. We may write $u_{j}=1+\sum_{i} \alpha_{j i} x_{i}+D_{j}$ where $D_{j}$ is in $\mathscr{J}^{2}$. Then

$$
\begin{aligned}
u_{j}^{q} & =\left(1+\sum \alpha_{j i} X_{i}+D_{j}\right)^{q}=1+\left(\sum \alpha_{j i} X_{i}+D_{j}\right)^{q} \\
& =1+\left(\sum \alpha_{j i} X_{i}\right)^{q}=1+\sum \alpha_{j i_{1}} \cdots \alpha_{j i_{q}} X_{i_{1}} \cdots X_{i_{q}}
\end{aligned}
$$

Received by the editors January 15, 1973.

AMS (MOS) subject classifications (1970). Primary 20F10, $20 \mathrm{E} 05$.

1 The author wishes to acknowledge the support of the National Science Foundation, and also the hospitality of the University of Birmingham.

(c) American Mathematical Society 1973 
summed over all $i_{1}, \cdots, i_{q}$ such that $1 \leqq i_{1}, \cdots, i_{q} \leqq n$. Therefore $u_{j}^{q M}=$ $1+M \sum \alpha_{j i_{1}} \cdots \alpha_{j i_{q}} X_{i_{1}} \cdots X_{i_{q}}$. It follows that

$$
u_{1}^{N} \cdots u_{m}^{N}=1+M \sum_{i_{1}, \cdots, i_{n}} \sum_{j=1}^{m} \alpha_{j i_{1}} \cdots \alpha_{j i_{q}} X_{i_{1}} \cdots X_{i_{q}} .
$$

Assume that (*) holds. Equating the coefficients of $X_{i}^{q}$ for each $i$ in (1) and (2) gives

$$
M=M \sum_{j=1}^{m} \alpha_{j i}^{a} \quad(1 \leqq i \leqq n) .
$$

Equating the coefficients of $X_{i}^{q-1} X_{h}$ for $i \neq h$ gives

$$
0=M \sum_{j=1}^{m} \alpha_{j i}^{\alpha-1} \alpha_{j h} \quad(1 \leqq i, h \leqq n ; i \neq h) .
$$

Since $p$ does not divide $M$, we may divide (3) and (4) through by $M$, obtaining

$$
\begin{gathered}
\sum_{j} \alpha_{j i}^{q}=1 \quad(1 \leqq i \leqq n), \\
\sum_{j} \alpha_{j i}^{q-1} \alpha_{j h}=0 \quad(1 \leqq i, h \leqq n ; i \neq h) .
\end{gathered}
$$

Let $A=\left(\alpha_{j i}^{q-1}\right)$ and $B=\left(\alpha_{j i}\right), m$-by- $n$ matrices over $G F(p)$. Then $\left(3^{\prime}\right)$ and $\left(4^{\prime}\right)$ assert that

$$
A^{T} B=I_{n}
$$

where $A^{T}$ is the transpose of $A$ and $I_{n}$ is the $n$-by-n identity matrix. It follows that $n=\operatorname{rank}\left(I_{n}\right) \leqq \operatorname{rank}(B) \leqq m$.

\section{REFERENCE}

1. Roger C. Lyndon and Morris Newman, Commutators as products of squares, Proc. Amer. Math. Soc. 39 (1973), 267-272.

Department of Mathematics, University of Michigan, Ann Arbor, Michigan 48104 (Current address of Roger Lyndon)

Départment de Mathematiques, Université de Montpellier, 34 Montpellier, France

Department of Mathematics, University College of Wales, Aberystwyth, Cardiganshire, Wales (Current address of Thomas McDonough)

National Bureau of Standards, Washington, D.C. 20234 (Current address of Morris Newman) 